\title{
ESTABILIDAD FENOTÍPICA DE CRUZAS SIMPLES E HÍBRIDOS COMERCIALES DE MAÍZ (Zea mays L.)
}

\section{PHENOTYPIC STABILITY OF SINGLE CROSSES AND COMMERCIAL HYBRIDS OF MAIZE (Zea mays L.)}

\author{
F. Javier Sánchez-Ramírez, Ma. del Carmen Mendoza-Castillo* \\ y C. Gabriela Mendoza-Mendoza
}

\begin{abstract}
Postgrado en Recursos Genéticos y Productividad-Genética, Campus Montecillo, Colegio de Postgraduados. Km 36.5 Carr. México-Texcoco. 56230, Montecillo, Texcoco, Edo. de México. Tel. 01 (595) 952-0200 Ext. 1524.

*Autor de correspondencia (camen@colpos.mx)
\end{abstract}

\section{RESUMEN}

Dada la diversidad de condiciones ambientales en las que se establece el cultivo de maíz (Zea mays L.) y la necesidad de genotipos mejorados de expresión consistente en las localidades, se evaluó la estabilidad fenotípica del rendimiento de grano, días a floración masculina y altura de planta de diez cruzas simples de maíz con rendimiento de grano sobresaliente, obtenidas mediante un esquema de cruzamientos dialélico con diez líneas $\mathrm{S}_{6}-\mathrm{S}_{8}$ y diez híbridos: comerciales de empresas locales y transnacionales y experimentales locales. El análisis de estabilidad se hizo mediante el modelo de efectos principales aditivos e interacción multiplicativa (AMMI), el cual se justificó mediante la significancia estadística de la interacción genotipo por localidad del análisis de varianza combinado. Las variables evaluadas respondieron de forma diferente a los ambientes, ya que los días a floración masculina y la altura de planta fueron las más afectadas; la estabilidad de los genotipos también difirió entre las características. Las cruzas simples fueron más estables que los híbridos comerciales, salvo para altura de planta; los híbridos mostraron interacción positiva con la localidad con mejores condiciones para la producción, pero en ningún caso con las que presentaron restricciones. Las cruzas, además de exhibir rendimiento de grano superior con respecto a algunos híbridos comerciales, tuvieron ciclos fenológicos más cortos y altura de planta similar a la que tienen los arquetipos comerciales actuales. Los resultados mostraron que las cruzas simples evaluadas pueden representar una opción para la producción de grano.

Palabras clave: Rendimiento de grano, días a floración masculina, altura de planta, interacción genotipo-localidad, AMMI.

\section{SUMMARY}

Given the diversity of environmental conditions under which maize (Zea mays $L$.) is sowed and the need to produce improved genotypes for consistent expression over locations, this research evaluated the phenotypic stability of grain yield, days to male flowering and plant height of ten simple crosses of maize outstanding by their grain yield. These crosses were obtained from a diallel crossing scheme with ten $\mathrm{S}_{6}-\mathrm{S}_{8}$ inbred lines, and ten hybrids: some of them commercially distributed by local and transnational companies and some others were local experimental hybrids. The stability analysis was performed by the additive main effects and multiplicative interactions (AMMI) model; this analysis was justified by the resulting statistical significance of the genotype - location interaction from the combined analysis of variance. The traits under evaluation were differentially modified by the environments (locations), being days to male flowering and plant height the most affected ones; the stability of the genotypes was also different between traits. Single crosses were more stable than commercial hybrids, except for plant height; hybrids displayed positive interaction with locations with better production conditions, but there was no interaction with locations with restrictive conditions. Aside from higher grain yield compared to some commercial hybrids, crosses had shorter phenological cycles and plant height similar to current commercial archetypes. Results showed that the single crosses evaluated might represent an option for maize grain production.

Index words: Grain yield, days to male flowering, plant height, interaction genotype by location, AMMI.

\section{INTRODUCCIÓN}

En México y alrededor del mundo, de acuerdo con Nzuve et al. (2013) el maíz (Zea mays L.) se cultiva en una amplia variedad de condiciones ambientales asociadas con el estado hídrico del suelo, radiación solar, temperatura, tipos de suelo y sistemas de producción; estas condiciones provocan expresiones diferenciadas del comportamiento de los genotipos, fenómeno denominado interacción genotipo por ambiente (McDermott y Coe, 2012), que representa una expresión constante en la mayoría de las características cuantitativas (Fan et al., 2007). Balzarini et al. (2005) señalaron que la interacción significativa conduce a dos situaciones: i) la estabilidad, que se refiere a la habilidad de los genotipos para expresarse de manera consistente y puede ser en ambos sentidos: rendimiento alto o bajo, y ii) la adaptación específica, descrita como la expresión sobresaliente de un genotipo en un determinado rango de ambientes o localidades.

En un programa de mejoramiento genético, el desarrollo de genotipos estables y con rendimiento alto es de fundamental importancia en la producción comercial de semilla, por tal motivo, en las etapas finales del proceso de mejoramiento, los genotipos desarrollados deben ser evaluados en diferentes localidades y durante varios ciclos, para identificar a aquellos con potencial sobresaliente antes de ser recomendados para cultivarse en alguna localidad o región (Tonk et al., 2011). 
La valoración de la estabilidad de un genotipo puede realizarse por diferentes métodos, dentro de los cuales se encuentra el análisis multivariado que tiene tres propósitos principales: i) eliminar el "ruido" en el conjunto de datos ya que discrimina entre variación sistemática y no sistemática, ii) sintetizar la información, y iii) mostrar la estructura o patrón de los datos (Crossa, 1990; Kandus et al., 2010).

El modelo de efectos principales aditivos e interacción multiplicativa (AMMI) es uno de los métodos de análisis de la estabilidad fenotípica más usados en la actualidad (Hongyu et al., 2014); para su interpretación se apoya en la representación gráfica usando "biplots" [representación gráfica mediante marcadores (vectores) para filas (genotipos) y columnas (localidades) de una matriz X] (Gabriel, 1971), ya que estos gráficos permiten observar de manera simultánea los genotipos (puntos) y los ambientes (vectores), así como la exploración de patrones de comportamiento atribuidos a los efectos de interacción genotipo por ambiente (Kandus et al., 2010; McDermott y Coe, 2012).

El AMMl es la combinación de una técnica estadística univariada (el análisis de varianza: ANAVA) con una técnica estadística multivariada (el análisis de componentes principales: ACP) (Zobel et al., 1988). Inicialmente, el AMMI ajusta los efectos principales aditivos, de genotipos y ambientes mediante el ANAVA y posteriormente, describe la parte no aditiva (interacción genotipo $\times$ ambiente $=\mid \mathrm{GA}$ ), mediante el ACP (Crossa, 1990; Zobel et al., 1988).

Una de las desventajas del AMMI se presenta cuando el número de componentes principales retenidos en el modelo es grande ( $\geq 3)$, ya que resulta difícil describir el comportamiento de los efectos de IGA por la imposibilidad de obtener gráficos en más de tres dimensiones, y aunque es posible graficar por pares, cada componente representará sólo una pequeña porción de la variación total de la interacción (Furtado et al., 2006).

Con base en lo anterior, además de que en México menos de $30 \%$ de la superficie sembrada con maíz se hace con semilla mejorada, y que una de las principales razones de esta baja proporción se debe a la falta de estabilidad o a la limitada adaptación específica de los híbridos comerciales, principalmente a las condiciones restrictivas de producción, se planteó valorar la estabilidad fenotípica del rendimiento de grano, de la floración masculina y la altura de planta en diez cruzas simples sobresalientes originadas de un sistema de cruzamientos dialélicos completo, y compararla con la expresión de diez híbridos (experimentales y comerciales) recomendados para los Valles Altos Centrales de México, para valorar el potencial del programa de mejoramiento y de las cruzas obtenidas.

\section{MATERIALES Y MÉTODOS}

Con un grupo de diez líneas endogámicas $\mathrm{S}_{6}-\mathrm{S}_{8^{\prime}}$, se desarrolló un esquema completo de cruzas dialélicas; las cuales junto con diez híbridos adicionales fueron evaluadas en un diseño experimental látice $10 \times 10$ con cuatro repeticiones y unidades experimentales de un surco de 5 $m$ de largo y 0.80 m entre éstos. La evaluación se realizó en tres localidades de los Valles Altos Centrales de México: Mixquiahuala, Hidalgo; Tecámac y Montecillo, Estado de México.

Durante el desarrollo del cultivo y en la cosecha se registraron 26 variables cuantitativas asociadas con rasgos morfológicos, fenológicos, rendimiento de grano y sus componentes con las cuales se realizó un análisis de varianza combinado (datos no mostrados). Dada la presencia de significancia estadística en la interacción genotipo x localidad para los diferentes rasgos, se hizo un análisis de estabilidad fenotípica con todos los genotipos mediante el cual se identificaron las diez cruzas simples sobresalientes por tener un rendimiento superior; posteriormente, éstas fueron comparadas con los híbridos.

De los 26 rasgos estudiados se eligió el rendimiento de grano, los días a floración masculina y la altura de planta como las principales características comparables en un genotipo mejorado, las cuales permitieron caracterizar el potencial de las cruzas simples con respecto a los híbridos.

El rendimiento de grano por unidad experimental $\left(\mathrm{RtO}_{\mathrm{UE}}\right)$ fue obtenido mediante la siguiente ecuación: Rto $_{\mathrm{UE}}=[(\mathrm{PMz} 1$ + PMz2) (Id) (100 - \% Hum)/100]/0.86; donde PMz1 y PMz2 corresponden al peso en campo por unidad experimental $(\mathrm{kg})$ de las mazorcas primarias y secundarias, respectivamente; Id, el índice de desgrane, obtenido de un promedio de cinco mazorcas, como la proporción entre el peso de grano y el peso de la mazorca; \% Hum, el porcentaje de humedad de la muestra en campo, obtenido a través del método de la estufa y un factor de corrección de 0.86, para ajustar la humedad del grano a $14 \%$.

Con el tamaño de la parcela $\left(4.0 \mathrm{~m}^{2}\right)$ se estimó el rendimiento de grano (Rto) en $\mathrm{t}$ ha ${ }^{-1}$. Los días a floración masculina (DFM) se determinaron cuando $50 \%+1$ de las plantas en la unidad experimental se encontraban en antesis. La altura de planta (Apta), medida en $\mathrm{cm}$ desde la base del suelo hasta la lígula de la hoja bandera, es el valor promedio de cinco plantas en cada unidad experimental.

El análisis de estabilidad se hizo empleando el modelo de efectos principales aditivos e interacción multiplicativa (AMMI): $Y_{i j}=\mu+g_{i}+e_{j}+\sum_{k=1}^{n} \lambda_{k} a_{i k} Y_{j k}+E_{i j}$ donde: $Y_{i j}=$ rendimiento del $i$-ésimo genotipo en el $j$-ésimo ambiente; $\mu=$ 
media general; $g_{i}=$ efecto del $i$-ésimo genotipo; $e_{j}=$ efecto del $j$-ésimo ambiente; $\lambda_{k}=$ raíz cuadrada del vector característico del $k$-ésimo eje del ACP; $a_{i k}=$ calificación del ACP para el $k$-ésimo eje del $i$-ésimo genotipo; $\gamma_{j k}=$ calificación del ACP para el $k$-ésimo eje del $j$-ésimo ambiente; $E_{i j}=$ valor del error.

El análisis de varianza combinado (no mostrado) se realizó con el paquete estadístico SAS, mientras que AMMI fue desarrollado con el "software" GEA-R (Pacheco et al., 2015), el cual emplea R.

Los "biplots" del modelo AMMI fueron elaborados mediante GEA-R y se encuentran representados en las Figuras 1 a 3 .

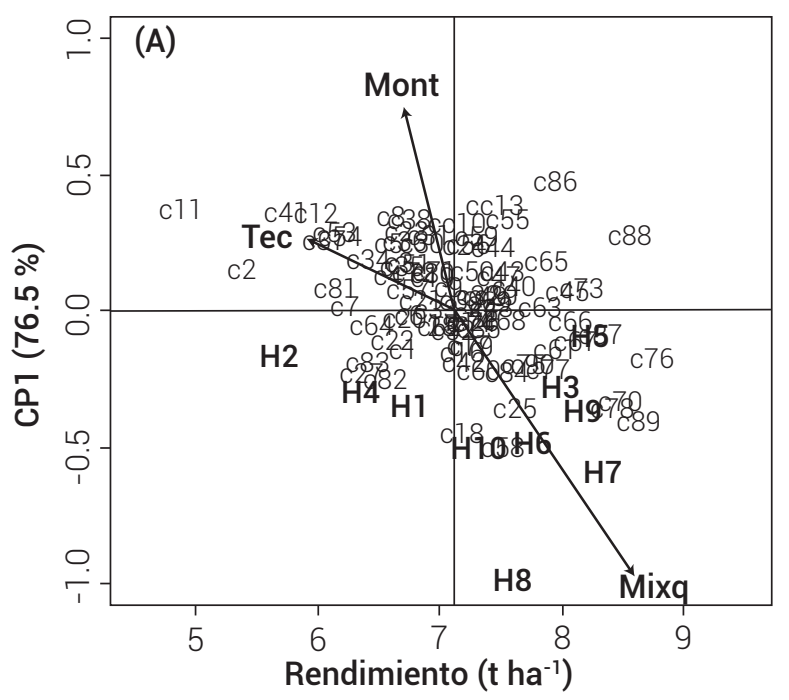

Las cruzas simples sobresalientes empleadas en la comparación fueron: c45, c66, c67, c70, c73, c76, c78, c87, c88, c89, y los híbridos: $\mathrm{H} 1$ y H2, experimentales; $\mathrm{H3}(\mathrm{H}-$ San José) y H4 (H-San Josecito), comerciales-locales; H5, $\mathrm{H} 6$ y H7, experimentales-mestizos y $\mathrm{H8}$ (H-Jabalí), $\mathrm{H9}(\mathrm{H}-$ Águila) y H10 (H-Caimán), comerciales-transnacionales.

\section{RESULTADOS Y DISCUSIÓN}

\section{Análisis AMMI}

En el Cuadro 1 se muestra el análisis de varianza de los efectos principales aditivos donde se encontraron diferencias significativas $(P \leq 0.01)$ para las variables estudiadas entre localidades y entre cruzas. La interacción Cruzas x Loc

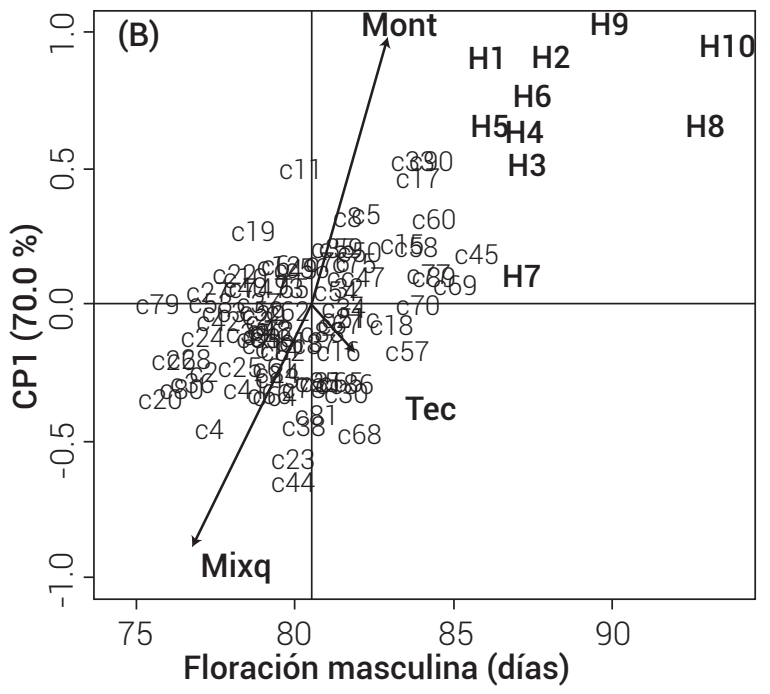

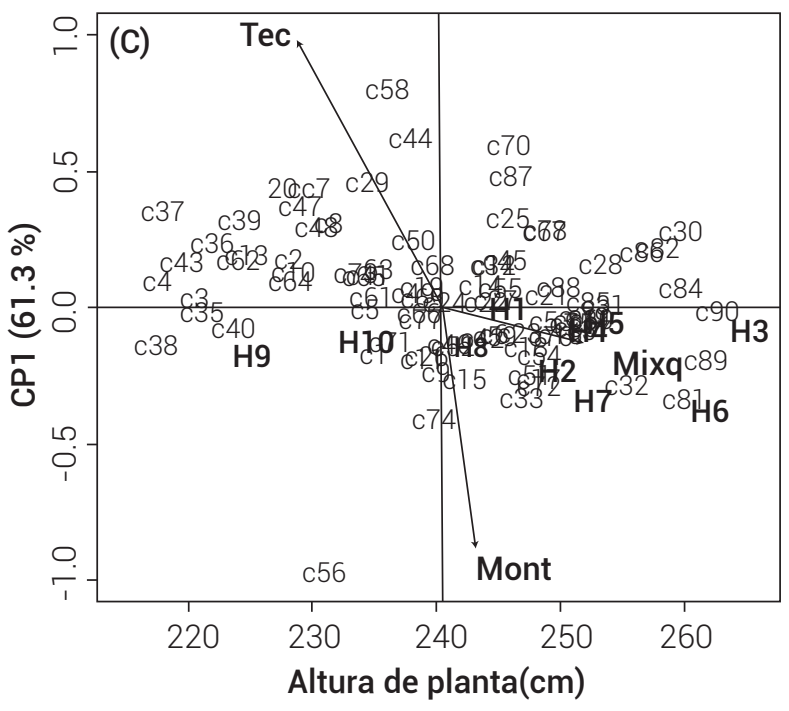

Figura 1. AMMI1. Interacción genotipo x localidad (CP1) y el valor medio del rendimiento de grano (A), días a floración masculina (B) y altura de planta (C) de cien genotipos (cruzas simples + híbridos) en tres localidades (Mont = Montecillo, Tec $=$ Tecámac y Mixq = Mixquiahuala) de los Valles Altos Centrales de México. 


Cuadro 1. Cuadrados medios del ANAVA del AMMI de tres variables cuantitativas obtenidas de 100
genotipos (cruzas simples + híbridos) evaluados en tres localidades de los Valles Altos Centrales de
\begin{tabular}{lcccc} 
México. \\
\hline FV & GL & Rto & DFM & Apta \\
\hline Localidades & 2 & $790.6 * *$ & $4384.7^{* *}$ & $45,423.6^{* *}$ \\
Cruzas & 99 & $6.7^{* *}$ & $137.4^{* *}$ & $167,063.9 * *$ \\
Cruzas x LOC & 198 & $3.62^{* *}$ & $9.6 * *$ & $42,633.9 * *$ \\
CP1 (\%) & & 76.5 & 70.0 & 61.3 \\
CP2 (\%) & 23.5 & 29.9 & 38.7 \\
CV (\%) & 17.92 & 3.0 & 5.2 \\
\hline
\end{tabular}

$\mathrm{FV}$ = factor de variación; $\mathrm{GL}$ = grados de libertad; Rto = rendimiento de grano; $\mathrm{DFM}=$ días a floración masculina; $A p t a=$ altura de planta; $\mathrm{CP} 1$ y CP2 = componente principal 1 y 2, respectivamente; $\mathrm{CV}=$ coeficiente de variación; $* *$ = significativo con $\mathrm{P} \leq 0.01$

fue estadísticamente significativa $(P \leq 0.01)$ e indicó que los genotipos no se comportaron de igual manera ante las variaciones ambientales de las diferentes localidades, lo cual justificó realizar el análisis de estabilidad.

Con base en la suma de cuadrados (datos no mostrados), la variación de cada variable se asoció con diferentes factores. La variación en Localidades fue mayor para el rendimiento de grano (53\%) y coincidió con lo señalado por Gauch y Zobel (1996) y Gauch (2006), quienes indicaron que en las evaluaciones multi-localidades para el rendimiento, la variación frecuentemente se concentra en el factor ambiental; no obstante, para DFM y Apta la variación se concentró en Cruzas (55 y $50 \%$, respectivamente), lo que denota amplia variación entre los genotipos estudiados (cruzas simples e híbridos). La interacción Cruzas $\mathrm{x}$ Loc tuvo diferentes porcentajes de variación entre las variables evaluadas, $24 \%$ en Rto, $8 \%$ en DFM y $13 \%$ en Apta, similares a los porcentajes obtenidos para rendimiento de grano por Tonk et al. (2011) y Nzuve et al. (2013), de $19 \%$ y $10.3 \%$, respectivamente.

De acuerdo con la prueba de Gollob (1968), la variación de la interacción Cruzas x Loc se explicó a través de los dos primeros componentes principales (Cuadro 1), lo cual se asoció con el número de localidades evaluadas, y aunque con los criterios de Gauch y Zobel (1996) el modelo AMMI fue altamente preciso, pues los dos primeros componentes principales explicaron la variación de la interacción; es necesario mencionar que la precisión del modelo disminuye conforme aumenta el número de localidades, tal como lo señalaron Lozano-Ramírez et al. (2015).

\section{Estabilidad fenotípica de cruzas simples e híbridos}

Con el total de los genotipos estudiados y para las tres variables evaluadas, se construyó el "biplot" AMMI1 (Figura 1) donde se encuentra el CP1 en el eje de las ordenadas y los efectos principales en el eje de las abscisas; la interacción genotipo ambiente se muestra de forma unidimensional (Gauch et al., 2008). Dada la dispersión de los puntos (genotipos), se observa que la interacción genotipo por localidad difiere entre las características evaluadas. La variable más afectada (mayor dispersión con respecto a la ordenada) por el efecto ambiental fue floración masculina (DFM), seguida por altura de la planta (Apta) y, no obstante que el rendimiento de grano (Rto) es un carácter genéticamente más complejo que los otros rasgos evaluados, éste fue relativamente más estable que el resto.

Dichos resultados se asociaron con la expresión contrastante de algunos genotipos por ejemplo: H10 mostró limitada interacción con el ambiente para Apta y una interacción muy alta para DFM e intermedia para Rto (Figura 3). Según Balzarini et al. (2005), este comportamiento puede corresponder con una limitada capacidad de adaptación específica de los genotipos. También pudiera deberse a los coeficientes de correlación entre rasgos, pues de acuerdo con Jugenheimer (1981), la altura de planta está correlacionada con los días a floración masculina (en este estudio fueron las características más afectadas por el ambiente), mientras que el rendimiento mostró correlación con la altura de planta, pero ésta fue baja.

Con base en lo anterior, es importante mencionar que los análisis de estabilidad se han realizado sólo con el rendimiento de grano (Fan et al., 2007; Hongyu et al., 2014; Lozano-Ramírez et al., 2015; Nzuve et al., 2013) aun cuando otras características complementarias fundamentales, como la floración, la altura de planta y los componentes del rendimiento, que inciden directamente sobre la expresión de éste, también son fuertemente afectados por el ambiente. Por ello sería importante considerar estas variables para explicar la expresión de cada genotipo, así como para determinar la estabilidad fenotípica del mismo, especialmente cuando el objetivo es la liberación comercial de las cruzas simples sobresalientes. 
Entre localidades la expresión de las características bajo estudio fue contrastante (Figura 1), debido a que hubo condiciones ambientales inesperadas. En Mixquiahuala las condiciones hídricas fueron inferiores a las comúnmente empleadas en el área agrícola, ya que de aplicar seis riegos sólo se llevaron a cabo tres: en presiembra, en floración y durante el llenado de grano, mismos que fueron suficientes para la producción de grano, dada la temperatura y la fertilidad del suelo favorables, lo cual puede corroborarse en la Figura 1 donde sobresale Mixquiahuala por tener el mayor rendimiento, ciclo más corto y plantas más altas.

En Montecillo, donde el ensayo se llevó a cabo bajo condiciones de cultivo cercanas a las óptimas, la incidencia de Fusarium spp. fue una limitante que provocó disminución significativa del rendimiento. En Tecámac, las condiciones hídricas fueron la principal condición restrictiva, pues sólo se aplicaron dos riegos de auxilio al cultivo, lo que causó que el rendimiento de grano y la altura de la planta fueran los menores entre las localidades. A pesar de estas condiciones ambientales, con el análisis empleado fue posible discriminar el comportamiento medio de los genotipos.

En la Figura 2, construida con AMMI2 (CP1 y CP2) se observa la interacción genotipo $x$ localidad para el rendimiento de grano de las cruzas simples sobresalientes y los híbridos; este modelo se emplea para determinar la asociación entre los genotipos y las localidades. Se encontró asociación positiva entre la localidad Mixquiahuala y los genotipos, principalmente los híbridos $\mathrm{H} 7, \mathrm{H} 8, \mathrm{H} 9$ y H10; el primero $(\mathrm{H} 7)$ es un híbrido experimental tipo mestizo y el resto son híbridos comerciales de empresas transnacio- nales. Las cruzas simples sobresalientes mostraron menor interacción con las localidades, lo que denota estabilidad a través de éstas. Aunque no hubo cruzas sobresalientes asociadas a Tecámac (la localidad más restrictiva), sí las hubo asociadas a Montecillo (c45, c73 y c88), lo que pudiera indicar cierta tolerancia a problemas fitopatológicos, aunque es necesario realizar dicha valoración ex profeso.

Con los resultados obtenidos se determinó que los híbridos, principalmente los de empresas transnacionales ( $\mathrm{H} 8$, H9 y H10), mostraron adaptación específica a la localidad con mejores condiciones para la producción de maíz; por tanto, aunque estos genotipos son una opción para ambientes con condiciones propicias para la producción de grano, no lo son para localidades con condiciones ambientales restrictivas. En cuanto a la estabilidad (Figura 2), las cruzas con expresión satisfactoria fueron c45, c66, c67 y c87, por haber sido más estables que la mayoría de los híbridos, con excepción de $\mathrm{H} 5$ que también fue estable, pues en su condición de mestizo se asoció con el probador de amplia base genética.

En la Figura 3 se muestra mediante el AMMI1, la interacción genotipo $x$ localidad (CP1) y el valor medio del rendimiento de grano (A), los días a floración masculina (B) y la altura de la planta (C) de las diez cruzas simples sobresalientes y los diez híbridos evaluados en tres localidades de los Valles Altos Centrales de México.

En la Figura 3 A que muestra el AMMI1 para rendimiento de grano, se puede apreciar el valor fenotípico (abscisa) y la estabilidad (ordenada) de las cruzas sobresalientes y

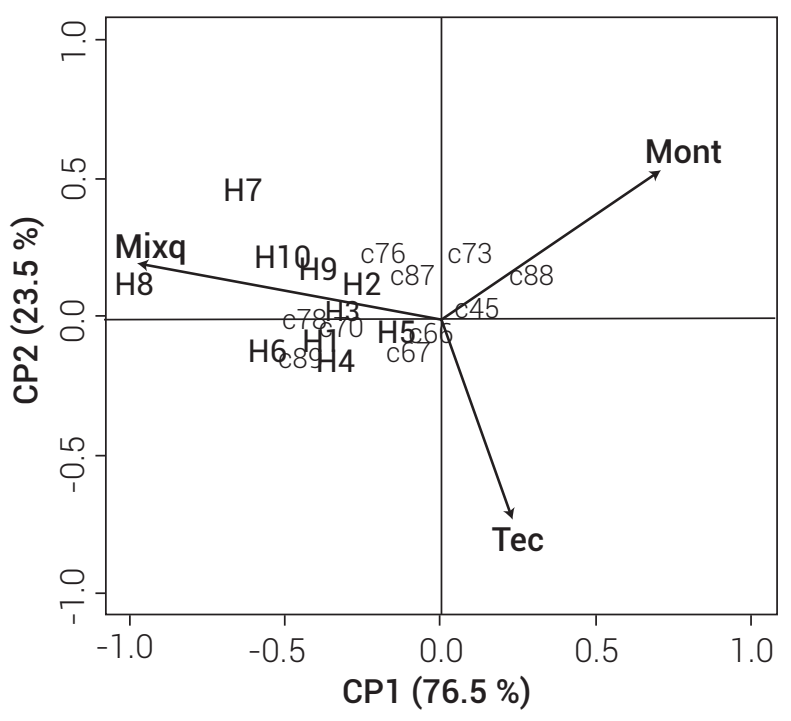

Figura 2. AMMI2. Representación gráfica del CP1 y CP2 mostrando la interacción genotipo x localidad de las diez cruzas simples sobresalientes y diez híbridos $(\mathrm{H} 1$ a H10), en tres localidades (Mont = Montecillo, Tec = Tecámac y Mixq = Mixquiahuala) de los Valles Centrales de México. 

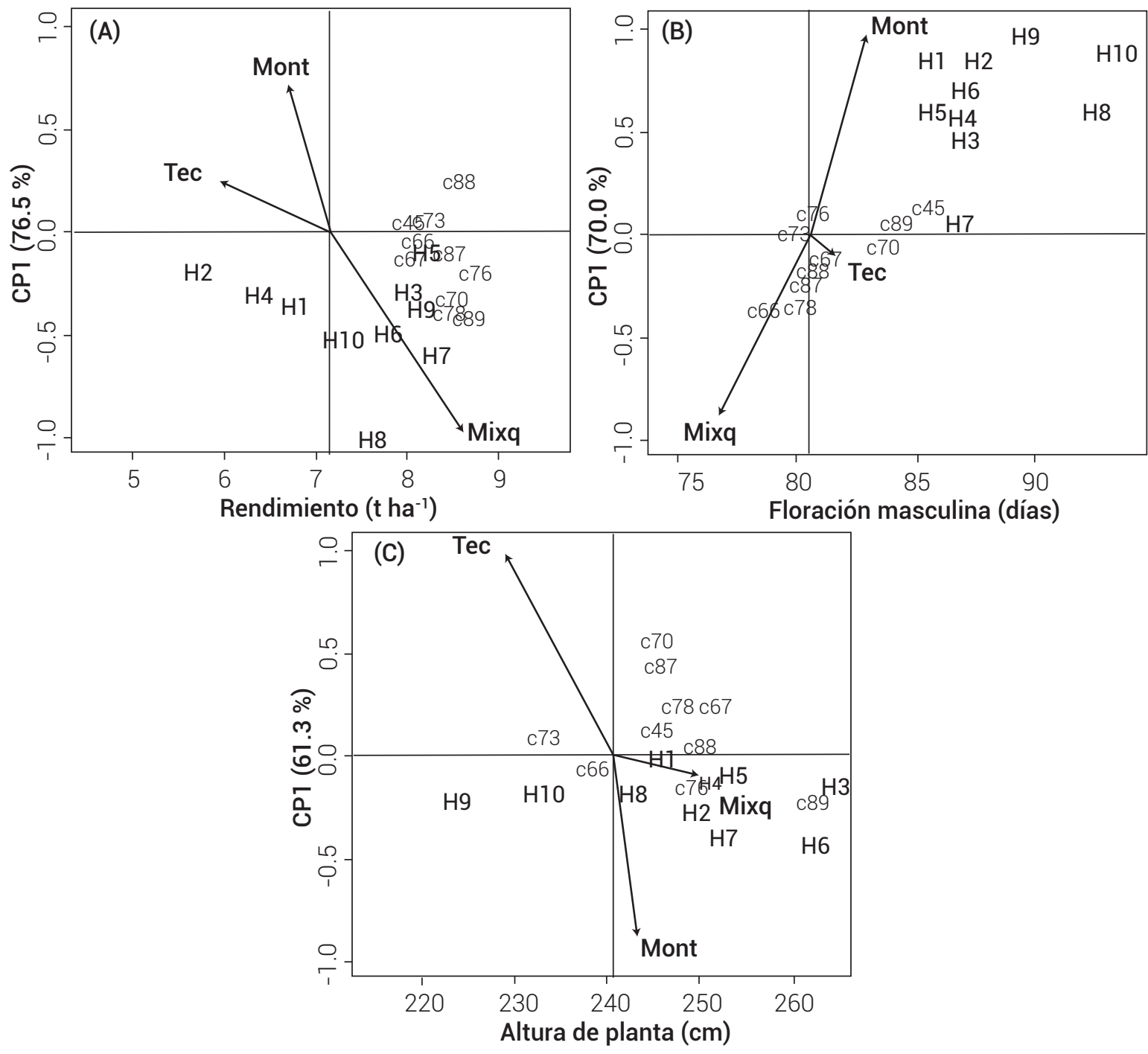

Figura 3. AMMI1. Interacción genotipo $x$ localidad (CP1) y valor medio del rendimiento de grano (A), días a floración masculina (B) y altura de planta (C) de diez cruzas simples y diez híbridos, evaluados en tres localidades (Mont = Montecillo, Tec = Tecámac y Mixq = Mixquiahuala) de los Valles Altos Centrales de México.

los híbridos. Entre localidades el rendimiento fue mayor en Mixquiahuala (8.7 t ha-1) y menor en Montecillo y Tecámac. Con respecto a los genotipos, el rendimiento de grano tuvo un intervalo de variación entre 5.5 y 9.0 t ha-1 $^{-1}$; algunas cruzas (c70, c76, c78, c87, c88 y c89) superaron al híbrido de mayor rendimiento $(\mathrm{H} 7)$; entre los testigos, tres produjeron más de $8.0 \mathrm{t} \mathrm{ha}^{-1}$, dos experimentales tipo mestizo ( $\mathrm{H} 5 \mathrm{y}$ $\mathrm{H7}$ ) y uno comercial transnacional (H9). Las cruzas sobresalientes por su estabilidad fueron c45, c66 y c73; sin embargo, al seleccionar por ambas cualidades (rendimiento de grano alto y estabilidad) se consideró que la mejor cruza fue c76, pues supera el rendimiento de todos los híbridos y la estabilidad de la mayoría, salvo H5.

La floración masculina (Figura 3 B) fue el rasgo más afectado por el ambiente; entre localidades, Mixquiahuala tuvo el ciclo fue más corto ( 77 d) que Tecámac y Montecillo (83 y 84 d, respectivamente), expresión que se vinculó con el efecto de la temperatura. Para esta característica los híbridos mostraron alta interacción genotipo por ambiente, excepto H7 (híbrido experimental mestizo), cuya respuesta también se relacionó con su amplia base genética; los DFM fluctuaron entre 78 y $95 \mathrm{~d}$; los híbridos mostraron un ciclo más largo que las cruzas, e incluso los tres híbridos comerciales de empresas transnacionales fueron tardíos. Se encontró una diferencia de al menos 5 d entre la cruza de mayor rendimiento (c76) y el híbrido más sobresaliente $(\mathrm{H} 7)$. Con base en esta información y tomando en cuenta los desfasamientos actuales del calendario agrícola con el cambio climático, se considera que las cruzas simples 
evaluadas presentan ventajas, no sólo por la expresión del rendimiento, sino además por mostrar menor DFM, comparadas con los híbridos estudiados.

La evaluación de Apta (Figura 3 C) mostró que el comportamiento de esta variable fue similar en Mixquiahuala y Montecillo, y ambas superiores que Tecámac; ello permite inferir que el déficit hídrico sufrido en esta localidad tuvo una limitada implicación en la altura de planta, que en este caso no se asoció con un ciclo precoz, probablemente debido al efecto térmico. Entre los genotipos, Apta presentó una variación de entre 225 y $265 \mathrm{~cm}$; tres genotipos, dos híbridos (H3, comercial local y H6, experimental mestizo) y una cruza simple sobresaliente (c89) tuvieron Apta mayor a $260 \mathrm{~cm}$, lo que es indeseable, aunque la mayoría de las cruzas presentaron Apta entre 240 y $250 \mathrm{~cm}$, intervalo que se consideró aceptable, de acuerdo con los arquetipos actuales disponibles en el mercado y para las condiciones del cultivo en el área agrícola.

La Apta fue la característica en la que los híbridos mostraron la menor interacción con el ambiente respecto a las cruzas sobresalientes; se observó además que aunque los híbridos H8, H9 y H10 presentaron DFM mayores, sus Apta fueron menores; es decir, que para los híbridos, especialmente los comerciales de empresas transnacionales, el efecto ambiental fue alto para DFM pero bajo para Apta. Con base en este resultado, vale la pena mencionar que la estabilidad de un genotipo también puede variar dependiendo del carácter considerado, lo cual posiblemente pueda depender de la correlación entre las características.

Al hacer la comparación entre la estabilidad del rendimiento de grano, los días a floración masculina y la altura de planta, entre diez cruzas sobresalientes y diez híbridos, se encontró que se tiene potencial para la generación de genotipos mejorados con capacidad de producción igual o superior a los híbridos comerciales actuales y con mayor capacidad de producción bajo condiciones ambientales restrictivas, con lo cual es posible incidir positivamente sobre la producción de maíz en los Valles Altos Centrales de México.

\section{CONCLUSIONES}

El efecto ambiental fue diferente entre las características cuantitativas evaluadas: rendimiento de grano, días a floración masculina y altura de planta, así también para los genotipos estudiados.

Las cruzas simples sobresalientes mostraron mayor estabilidad con respecto a los híbridos comerciales o experimentales; con algunas cruzas se logró una mejor expresión fenotípica. Los híbridos, principalmente los comerciales de empresas transnacionales, mostraron afinidad para la producción en localidades con condiciones favorables mientras que las cruzas sobresalientes se asociaron tanto a condiciones favorables de cultivo como a condiciones restrictivas.

\section{BIBLIOGRAFÍA}

Balzarini M., C. Bruno y A. Arroyo (2005) Análisis de Ensayos Agrícolas Multi-ambientales. Ejemplos con Info-Gen. Facultad de Ciencias Agropecuarias. Universidad Nacional de Córdoba. Córdoba Argetina $141 \mathrm{p}$

Crossa J. (1990) Statistical analyses of multilocation trials. Advances in Agronomy 44:55-85.

Fan X. M., M. S. Kang, H. Chen, Y. Zhang, J. Tan and C. Xu (2007) Yield stability of maize hybrids evaluated in multi-environment trials in Yunnan, China. Agronomy Journal 99:220-228.

Furtado F. D., C. García B. D., B. F. John M., A. Almeida M. and R. Vencovsky (2006) Statistical model in agriculture: biometrical methods for evaluating phenotypic stability in plant breeding. Cerne Lavras 12:373-388.

Gabriel K. R. (1971) The biplot graphic display of matrices with application to principal component analysis. Biometrika 58:453-467.

Gauch H. G. (2006) Statistical analysis of yield trials by AMMI and GGE. Crop Science 46:1488-1500

Gauch H. G. and R. W. Zobel (1996) AMMI analysis of yield trials In: Genotype by Environment Interaction. M.S. Kang and H. G. Gauch Jr. (eds.). CRC Press. Boca Raton Florida. pp 85-122.

Gauch H.G., H. P. Piepho and P. Annicchiarico (2008) Statistical analysis of yield trials by AMMI and GGE: further considerations. Crop Science 48:866-889.

Gollob H. F. (1968) A statistical model which combines features of factor analytic and analysis of variance techniques. Psychometrika 33:73-115

Hongyu K., M. García Peña, L. Borges de Araújo and C. T. dos Santos Dias (2014) Statistical analysis of yield trials by AMMI analysis of genotype environment interaction. Biometrical Letters 51:89102.

Jugenheimer R. W. (1981) Maíz: Variedades Mejoradas, Métodos de Cultivo y Producción de Semillas. Ed. Limusa. México, D. F. 841 p.

Kandus M., D. Almorza, R. Boggio R. and J. C. Salerno (2010) Statistical models for evaluating the genotype-environment interaction in maize (Zea mays L.). ФYTON. International Journal of Experimental Botany 79:39-46

Lozano-Ramírez A., A. Santacruz-Varela, F. San-Vicente-García, J. Crossa, J. Burgueño y J. D. Molina-Galán (2015) Modelación de la interacción genotipo $\times$ ambiente en rendimiento de híbridos de maíz blanco en ambientes multiples. Revista Fitotecnia Mexicana 38:337347.

McDermott B. and R. Coe (2012) An Easy Introduction to Biplots for Multienvironment Trials. Statistical Services Center, University of Reading, UK and World Agroforestry Centre, Kenya. 62 p.

Nzuve F., S. Githiri, D. M. Mukunya and J. Gethi (2013) Analysis of genotype $\times$ environment interaction for grain yield in maize hybrids. Journal of Agricultural Science 5:75-85.

Pacheco A., M. Vargas, G. Alvarado, F. Rodríguez, J. Crossa and J. Burgueño (2015) GEA-R (Genotype Environment Analysis with R for Windows) Version 2.0. International Maize And Wheat Improvement Center. http://hdl.handle.net/11529/10203 (Diciembre, 2015).

Tonk A. F., E. Ilker and M. Tosun (2011) Evaluation of genotype environment interactions in maize hybrids using GGE biplot analysis. Crop Breeding and Applied Biotechnology 11:1-9

Zobel R. W., M. J. Wright and H. G. Gauch Jr. (1988) Statistical analysis of yield trial. Agronomy Journal 80:388-393. 